\title{
OPTIMISATION OF RECURRENT SELECTION UNDER MUTAGENESIS IN MICRO-ORGANISMS
}

\author{
M. SOLLER \\ Department of Genetics, The Hebrew University of Jerusalem
}

Received 2.x.79

\begin{abstract}
Summary
A general model is presented for the response of a haploid population reproducing asexually to recurrent cycles of mutagenesis and selection. It is concluded that the determinants of genetic progress in such populations differ from those in sexually reproducing populations in that (1) measurement errors are relatively unimportant in impeding progress, and (2) the proportion selected has a powerful effect on genetic variance in future generations. Dividing available testing resources between two selection lines, with two or three cycles of hybridisation and selection every five to ten cycles of mutagenesis can increase gains if numerous loci can potentially mutate to plus alleles, and if the variance induced by mutation is not very much less than the error variance. Programmes involving hybridisation become more attractive in the presence of a deleterious effect of mutagenesis on mean population value. Three conditions for the success of recurrent selection programmes based on mutagenesis are (1) the production by mutagenesis of a modicum of positive genetic changes, (2) the absence of strong effects of mutagenesis on the mean value of the population, and (3) the absence of strong genotype $\times$ testenvironment interactions. The exact shape of the genetic distribution following mutagenesis, the total number of mutable loci and the presence of epistatic interactions between successive mutations are less critical.
\end{abstract}

\section{INTRODUGTION}

Mutagenesis has been applied with conspicuous success to the improvement of production traits in industrial micro-organisms, the basic approach being a search, using appropriate screening procedures, for stepwise mutations strongly affecting the production trait (Sermonti, 1969). Although such major mutational steps are rare, mutagenic treatment is also known to produce a relatively large number of minor genetic changes affecting the trait, giving rise to a mutagenised population showing a continuous spectrum of genetic variation (Davies, 1964; Dulaney and Dulaney, 1967; Alikhanian, 1970). Davies (1964) pointed out that this type of genetic variation could be exploited for purposes of genetic improvement by the application of repeated cycles of mutagenesis and selection to a microbial population. Making some assumptions about the distribution of this genetic variation and utilising Monte Carlo methods, he also evaluated the effects of various biological and technical parameters on population response. The purpose of the present study is to develop a more general model, based on classical quantitative genetic theory (Falconer, 1960), for predicting the response of a microbial population to recurrent cycles of mutagenesis and selection. Since methods are available for sexual or parasexual hybridisation in many microbial species (Burnett, 1975), the response 
to selection in progenies of crosses between lines developed under mutagenesis are also considered. The model is then utilised to identify some of the biological and technical parameters whose values are critical to the success and optimisation of such selection programmes.

\section{THEORY}

\section{(i) The response to recurrent cycles of mutagenesis and selection}

We consider an initial population derived asexually from a single clone with mean value, $\mu$, for some particular quantitative trait. Measurement errors are assumed to be normally distributed with mean, 0 , and variance $\sigma_{e}^{2}$. Between each round of selection, spontaneous and induced mutations affecting the quantitative trait accumulate, producing at the selection step a population with a distribution of genetic values having mean $\mu_{m}=\mu+d$ and variance $\sigma_{m}^{2}$. It is assumed that $d$ and $\sigma_{m}^{2}$ remain constant from cycle to cycle. It should be noted that $d$ will often be negative in value, as a result of a negatively skewed post-mutagenic genetic distribution, or of a general deleterious effect of mutagenesis on loci affecting vigour and viability. In the selection step it is assumed that a total of $\mathcal{N}$ colonies are scored for the quantitative trait, and the best $n$ are selected to serve as progenitors of the population subjected to the next round of mutagenesis. This process is repeated for a given number of cycles of mutagenesis and selection, or until it ceases to yield additional gains.

Genetic gains per cycle under this procedure can be calculated as follows. At the first selection step the only source of genetic variation is mutation, so that total genetic variance, $\sigma_{g}^{2}$, equals $\sigma_{m}^{2}$. In the subsequent cycles, however, there are two sources of genetic variation: $\sigma_{m}^{2}$, due to mutagenesis, and $\sigma_{w}^{2}$, due to genetic differences among the $n$ parental colonies chosen in the previous cycle. Thus, after the $j$ th cycle of mutagenesis and selection, total genetic variance, $\sigma_{g}^{2}(j)$, will equal

$$
\sigma_{g(j)}^{2}=\sigma_{m}^{2}+\sigma_{w(j-1)}^{2}
$$

and total phenotypic variance of measured values of the colonies of the $j$ th cycle, $\sigma_{j}^{2}$ equal

$$
\sigma_{j}^{2}=\sigma_{g(j)}^{2}+\sigma_{e}^{2}
$$

In the $j$ th cycle, the genetic variance, $\sigma_{w(j)}^{2}$, between $n$ selected colonies will arise from two sources,

(i) Differences in expected genetic values of selected colonies having different phenotypic values, and

(ii) Differences in the genetic values of individual selected colonies having the same phenotypic value.

Following Soller and Genizi (1975), (ii) will equal

$$
\frac{\sigma_{g(j)}^{2}}{\sigma_{j}^{2}}\left(1-\frac{\sigma_{g(j)}^{2}}{\sigma_{j}^{2}}\right) \sigma_{j}^{2}
$$

while (i) can be calculated as follows: The variance of phenotypic values of the selected colonies will equal $\lambda \sigma_{j}^{2}$, where $\lambda=1-i(i-x)$ is the proportion of the phenotypic variance remaining in the selected colonies, $i$ is the 
standardised unit normal selection differential, and $x$ is the normal point of truncation. By classical quantitative genetic theory (Falconer, 1960) the expected genetic value of a colony having phenotypic value, $y-\mu$, will be $\frac{\sigma_{\theta}^{2}}{\sigma^{2}}(y-\mu)$. Hence the variance of expected genetic values of $n$ selected colonies will be

$$
\left(\frac{\sigma_{g(j)}^{2}}{\sigma_{j}^{2}}\right)^{2} \sigma_{j}^{2}
$$

Summing (i) and (ii), simplifying and multiplying by $\frac{n-1}{n}$ to account for the reduction in population genetic variance due to finiteness of the selected sample, we have

$$
\sigma_{w(j)}^{2}=\sigma_{g(j)}^{2}\left(\frac{n-1}{n}\right)\left[1-(1-\lambda) \frac{\sigma_{g(j)}^{2}}{\sigma_{j}^{2}}\right]
$$

The recurrence relation for $\sigma_{\theta(j)}^{2}$ now becomes

$$
\sigma_{g(j)}^{2}=\sigma_{m}^{2}+\sigma_{g(j-1)}^{2}\left(\frac{n-1}{n}\right)\left[1-(1-\lambda) \frac{\sigma_{g(j-1)}^{2}}{\sigma_{(j-1)}^{2}}\right]
$$

Since $\left(\frac{n-1}{n}\right)\left[1-(1-\lambda) \frac{\sigma_{g(j-1)}^{2}}{\sigma_{(j-1)}^{2}}\right]$ will be less than 1 , say $\beta_{j}$, the recurrence relation for $\sigma_{g(j)}^{2}$ has the form

$$
\sigma_{g(j)}^{2}=\sigma_{m}^{2}+\sigma_{g(j-1)}^{2} \beta_{j}=\sigma_{m}^{2}\left(1+\beta_{1}+\beta_{1} \beta_{2}+\beta_{1} \beta_{2} \beta_{3}+\ldots+\beta_{1} \beta_{2} \ldots \beta_{j}\right)
$$

which will more or less rapidly approach an upper limit at which

$$
\sigma_{g(j)}^{2}=\sigma_{g(j-1)}^{2}
$$

Setting $\sigma_{g(j)}^{2}=\sigma_{g(t)}^{2}$ at the limit, taking $\sigma_{t}^{2}=\sigma_{g(t)}^{2}+\sigma_{e}^{2}$, and rearranging terms gives a quadratic equation in $\sigma_{g(t)}^{2}$, with the solution

$$
\sigma_{g(t)}^{2}=\frac{\sigma_{m}^{2}-\frac{\sigma_{e}^{2}}{n}+\sqrt{\left(\sigma_{m}^{2}-\frac{\sigma_{e}^{2}}{n}\right)^{2}+4\left[1-\frac{(n-1) \lambda}{n}\right] \sigma_{e}^{2} \sigma_{m}^{2}}}{2\left[1-\frac{(n-1) \lambda}{n}\right]}
$$

On the above assumptions, and on usual quantitative genetic theory (Falconer, 1960), genetic gains, $\Delta G_{m}$, per selection step will be

$$
\Delta G_{m}=\sigma_{g(t)} h_{(t)} i
$$

where, $\sigma_{g}$ and $i$ are as before, and $h_{(t)}=\sigma_{g(t)} / \sigma_{(t)}$ is the correlation between genotype and phenotype at limit values of $\sigma_{g}$. Values of $i$ for various $n$ and $\mathcal{N}$ assuming normality were obtained from Becker (1967). The effect of deviations from normality will be considered in the REsults section.

Since selection takes place after mutagenesis, genetic gains in population values as a result of selection, refer back to the population mean after mutagenesis. Consequently, net change in mean population value as a result of repeated cycles of mutagenesis and selection, $\Delta G_{n e t}$, will depend on the 
relative magnitudes of the effect of mutagenesis on mean population value on the one hand $(d)$, and the genetic gains within the mutagenised population due to selection on the other, i.e.,

$$
\Delta G_{\mathrm{net}}=\Delta G_{m}+d
$$

(ii) The response to selection in populations formed by hybridisation of lines developed under mutagenesis

Assuming that mutations at many loci can affect a given quantitative trait, two lines subjected to identical degrees of mutagenesis and selection, and showing about the same amount of genetic change, might yet have accumulated plus alleles at basically different sets of loci. In such circumstances, hybridising the two lines and selecting among the recombinant progeny might yield genetic gains without requiring additional cycles of mutagenesis. This might serve as a particularly potent means of genetic improvement when mutagenesis has a negative effect on mean population value. In this section we evaluate one possible design for the exploitation of this mode of genetic improvement. The design we consider is to develop simultaneously and independently, two mutagenesis-selection lines, with half of the available testing facilities devoted to the development of each line. After a number of cycles of mutagenesis and selection, superior colonies in each line, chosen at the last mutagenesis cycle, would be hybridised at random and selection carried out among the recombinant progeny. Hybridisation and selection cycles would be repeated until fixation was approached for most of the plus alleles. At this point the population could be split again into two lines and a new series of mutagenesis-selection cycles carried out within each line.

\section{(a) Total expected response during the hybridisation and selection cycles}

The total response expected under the hybridisation-selection cycles can be evaluated analytically under the following assumptions: (1) each cycle of mutagenesis and selection results in the accumulation in each line of an additional plus allele of average value $a_{r}$, where $a_{r}$ is the mean genetic gain per cycle in each line at limit values of $\sigma_{g}$ and $h$, and optimum values of $n$, and (2) within each line, the accumulated mutations are independent and wholly additive in their effects. That is, after $t_{m}$ cycles of mutagenesis and selection each line is assumed to have accumulated plus alleles of average value $a_{r}$ at each of $t_{m}$ loci, for a total response, including effects of mutagenesis on the population mean, of $t_{m}\left(a_{r}+d\right)$.

Hybridisation and selection allow all of these plus alleles to be collected in a single recombinant population. In contrast, since there is no selection for deleterious effects, $d$, at most, would remain at its final level in the individual mutagenesis selection lines prior to hybridisation, namely, $t_{m} d$. Thus, the total gain in value in the hybridised population, relative to the original starting population, would be $t_{m}(2-v) a_{r}+t_{m} d=t_{m}\left\{(2-v) a_{r}+d\right\}$, where $v$ is the proportion of overlapping mutations. Subtracting from this the response during the mutagenesis cycles yields $t_{m}(1-v) a_{r}$ as the maximum additional gains in the recombinant population as a result of $t_{r}$ cycles of hybridisation and selection. It should be noted that this expression is independent of $d$. This is a reflection of the fact that hybridisation allows 
additional plus alleles to be incorporated into the line, without the necessity for additional mutagenesis treatments. Thus when the effect of mutagenesis on the population mean is deleterious and of the same order of magnitude as $a_{r}$, response during the mutagenesis cycles might be minimal or even negative, while response during the hybridisation cycles could be strongly positive.

\section{(b) Relative response in a combined mutagenesis and hybridisation programme compared to a programme based on mutagenesis alone}

In comparing relative response in a combined programme to that based on mutagenesis alone, it should be noted that under mutagenesis alone all of the testing facilities are concentrated on a single line, and hence the proportion selected will be $n$ out of $\mathcal{N}$, while in the combined programmes testing facilities are divided among two lines with a proportion selected of $n$ out of $\mathcal{N} / 2$ in each line. For most values of $n$ and $\mathcal{N}$ the selection differential, $i_{m}$, for the best $n / \mathcal{N}$ will be significantly greater than the selection differential, $i_{r}$, for the best $n / \frac{1}{2} \mathcal{N}$. Thus, $a_{m}$, the average value of the plus alleles accumulated in a programme based on mutagenesis only, will be greater than $a_{r}$, and equal to $a_{r} i_{m} / i_{r}$.

Relative response can now be approximated as follows. Total response in the combined programme over the $t_{m}$ mutagenesis cycles and $t_{r}$ hybridisation cycles will be $t_{m}\left\{(2-v) a_{r}+d\right\}$, as above. Total response over the same total number of cycles in a mutagenesis programme would be $\left(t_{m}+t_{r}\right)$ $\left(a_{m}+d\right)$. Relative response will therefore be

$$
\frac{t_{m}\left\{(2-v) a_{r}+d\right\}}{\left(t_{m}+t_{r}\right)\left(a_{m}+d\right)}
$$

Consideration of this expression shows that relative response will be greatest when $v$ is small (i.e., many loci affect the trait), when $d$ is negative (i.e., mutagenesis has a deleterious effect on mean population value) and when $t_{m}$ is large relative to $t_{r}$. Under the converse conditions, particularly in view of the fact that $a_{m}>a_{r}$, response in the combined programme can readily be less than under mutagenesis alone.

The number of hybridisation-selection cycles, $t_{r}$, is itself a function of the number of mutagenesis cycles, in that the latter determines the number of plus alleles accumulated in each mutagenesis line, and hence the total recombinational genetic variance released upon hybridisation. Clearly, the greater this total, the greater the number of hybridisation-selection cycles required for its full exploitation. In practice, however, hybridisationselection cycles would not be continued until exhaustion of the recombinational genetic variance, but only to the point at which anticipated genetic gains from an additional hybridisation-selection cycle, $\Delta G_{r}$, will be less than those from an additional mutagenesis-selection cycle, $\Delta G_{m}$. On this principle response in the combined programme was calculated as follows:

$$
\Delta G_{r(i)}=\sigma_{g r(i)} h_{r(i)} i
$$

where, $i$ is the unit normal selection differential

$\sigma_{g r(i)}^{2}$ is the genetic variance released by recombination in the $i$ th hybridisation cycle ${ }_{j}$ and will equal $2 t_{m}(1-v) p_{i} q_{i} a_{r}^{2}$, where the term 
$2 t_{m}(1-v)$ represents the number of segregating loci in the initial hybrid population and $p_{i}$ and $q_{i}$ are the frequencies of plus and minus alleles, respectively.

$h_{r(i)}$ is the correlation between genotype and phenotype in the $i$ th hybridisation cycle, and will equal $\sigma_{g r(i)} / \sigma_{r(i)}$, where $\sigma_{r(i)}^{2}=\sigma_{g r(i)}^{2}+\sigma_{e}^{2}$ is the total phenotypic variance of measured colony values in the $i$ th hybridisation cycle.

Gene frequencies in the $i$ th hybridisation cycle were obtained by successive applications of the following expression (based on Falconer, 1960, p. 205) for the change in gene frequency in the $i$ th cycle, $\Delta p_{i}$,

$$
\Delta p_{i}=p_{i} q_{i} a_{r} i / \sigma_{r}
$$

and assuming $p=q=0.5$ in the initial hybrid population.

Relative progress under a combined programme of mutational-selection cycles followed by recombinational-selection cycles were calculated for $\sigma_{e}^{2}=9,4$ and 1, and assuming $n=5, \mathcal{N}=200$. Hybridisation selection cycles were terminated at the point where $\Delta G_{r(i)}<\Delta G_{m}+d$. Mean genetic gains per cycle over the hybridisation cycles were calculated by $\sum_{i=1}^{t_{r}} \Delta G_{r(i)} / t_{r}$ and compared to mean gains per mutagenesis cycle in the combined programme. Similarly, mean genetic gains over both mutagenesis hybridisation cycles of the combined programme were compared to mean gains in the programme based on mutagenesis alone.

\section{Results}

\section{(i) Response to recurrent selection under mutagenesis alone}

Table 1 shows limit values calculated by (2) for the genetic variation, $\sigma_{g(t)}$, and the correlation between genotype and phenotype $h_{t}$, for a quantitative trait in an asexual population under repeated cycles of mutagenesis and

\section{TABLE 1}

Limit values for the genetic variance, $\sigma_{g}^{2}(t)$, and for the correlation between genotype and phenotype, $h_{t}$, of a quantitative trait under repeated cycles of mutagenesis and selection, according to error variance, $\sigma_{e}^{2}$ and the number of colonies selected each cycle. Genetic and error variances in units of the variance induced by mutagenesis, $\sigma_{m}^{2}$

\begin{tabular}{|c|c|c|c|c|c|c|}
\hline \multirow{2}{*}{$\begin{array}{c}\text { Colonies } \\
\text { selected } \\
\text { per cycle } \\
(n)\end{array}$} & \multicolumn{2}{|c|}{$\sigma_{e}^{2}=9 \cdot 0$} & \multicolumn{2}{|c|}{$\sigma_{e}^{2}=4 \cdot 0$} & \multicolumn{2}{|c|}{$\sigma_{e}^{2}=1 \cdot 0$} \\
\hline & $\sigma_{g(t)}^{2}$ & $h_{t}$ & $\sigma_{g(t)}^{2}$ & $h_{t}$ & $\sigma_{g(t)}^{2}$ & $h_{t}$ \\
\hline 1 & $1 \cdot 00$ & $0 \cdot 32$ & $1 \cdot 00$ & 0.45 & $1 \cdot 00$ & $0 \cdot 71$ \\
\hline 2 & $1 \cdot 74$ & $0 \cdot 40$ & 1.59 & 0.53 & $1 \cdot 32$ & 0.75 \\
\hline 3 & $2 \cdot 22$ & 0.44 & 1.90 & 0.57 & $1 \cdot 45$ & $0 \cdot 77$ \\
\hline 4 & $2 \cdot 52$ & 0.47 & $2 \cdot 09$ & 0.59 & $1 \cdot 53$ & $0 \cdot 78$ \\
\hline 5 & $2 \cdot 74$ & $0 \cdot 48$ & $2 \cdot 21$ & $0 \cdot 60$ & $1 \cdot 58$ & $0 \cdot 78$ \\
\hline 6 & $2 \cdot 90$ & 0.49 & $2 \cdot 30$ & $0 \cdot 60$ & $1 \cdot 62$ & 0.79 \\
\hline 7 & $3 \cdot 02$ & 0.50 & $2 \cdot 37$ & $0 \cdot 61$ & $1 \cdot 64$ & $0 \cdot 79$ \\
\hline 8 & $3 \cdot 12$ & 0.51 & $2 \cdot 43$ & 0.61 & $1 \cdot 67$ & $0 \cdot 79$ \\
\hline 9 & $3 \cdot 20$ & 0.51 & $2 \cdot 47$ & $0 \cdot 62$ & $1 \cdot 69$ & $0 \cdot 79$ \\
\hline 10 & $3 \cdot 26$ & 0.52 & $2 \cdot 51$ & $0 \cdot 62$ & $1 \cdot 70$ & $0 \cdot 79$ \\
\hline 20 & $3 \cdot 62$ & 0.54 & $2 \cdot 71$ & $0 \cdot 64$ & $1 \cdot 80$ & 0.80 \\
\hline
\end{tabular}


selection. It is assumed that 200 colonies are tested each cycle, and values are given according to the relative magnitude of the error variance and the number of colonies selected each cycle $(n)$. Reiterations using (1) showed that limit values were closely approached in about six cycles. Limit values (not shown) were calculated for $\mathcal{N}=50$ and for $\mathcal{N}=1000$, and were virtually identical to those for $\mathcal{N}=200$. The outstanding result of table 1 is the increase in genetic standard deviation and in the correlation between genotype and phenotype with increasing $n$. This increase was greatest when error variance was relatively large.

Table 2 shows expected genetic gains calculated by (3) per selection step for a quantitative trait under repeated cycles of mutagenesis and selection, according to error variance, the number of colonies selected each cycle $(n)$, and the total number of colonies tested each cycle $(\mathcal{N})$. Limit

\section{TABLE 2}

Expected gains per cycle of selection for a quantitative trait under repeated cycles of mutagenesis and selection, according to error variance, $\sigma_{e}^{2}$, the number of colonies selected each cycle, $n$, and the total number of colonies tested each cycle, $\mathcal{N}$. Genetic gains and error variances given in units of the standard deviation and variance induced by mutagenesis, respectively

\begin{tabular}{|c|c|c|c|c|c|}
\hline $\begin{array}{l}\text { Colonies } \\
\text { selected }\end{array}$ & & $\sigma_{e}^{2}=9 \cdot 0$ & & $\sigma_{e}^{2}=4 \cdot 0$ & $\sigma_{e}^{2}=1 \cdot 0$ \\
\hline (n) & $\mathcal{N}=50$ & $\mathcal{N}=200$ & $\mathcal{N}=1000$ & $\mathcal{N}=200$ & $\mathcal{N}=200$ \\
\hline 1 & 0.71 & 0.87 & 1.06 & 1.23 & 1.94 \\
\hline 2 & $1 \cdot 10$ & $1 \cdot 37$ & 1.68 & $1 \cdot 74$ & $2 \cdot 24$ \\
\hline 3 & 1.28 & 1.63 & $2 \cdot 01$ & 1.93 & $2 \cdot 29$ \\
\hline 4 & 1.36 & 1.76 & $2 \cdot 19$ & $2 \cdot 01$ & $2 \cdot 28$ \\
\hline 5 & 1.39 & 1.84 & $2 \cdot 29$ & $2 \cdot 04$ & $2 \cdot 26$ \\
\hline 6 & 1.40 & 1.88 & 2.35 & 2.05 & $2 \cdot 23$ \\
\hline 7 & $1 \cdot 39$ & 1.90 & $2 \cdot 39$ & $2 \cdot 04$ & $2 \cdot 20$ \\
\hline 8 & $1 \cdot 37$ & 1.91 & $2 \cdot 42$ & $2 \cdot 04$ & $2 \cdot 17$ \\
\hline 9 & $1 \cdot 35$ & 1.91 & $2 \cdot 43$ & $2 \cdot 02$ & $2 \cdot 14$ \\
\hline 10 & $1 \cdot 33$ & 1.90 & $2 \cdot 44$ & 2.00 & $2 \cdot 11$ \\
\hline 20 & 1.05 & 1.77 & $2 \cdot 39$ & 1.82 & 1.87 \\
\hline
\end{tabular}

values for $\sigma_{g(t)}$ and $h_{t}$ were used in the calculations, and results are given in units of $\sigma_{m}$. It is assumed that mutagenesis has no effect on mean population value $(d=0)$ and that genotype $x$ test-environment interactions are absent. Depending on $\sigma_{e}^{2}$ and $\mathcal{N}$ there are clear, but rather broad optima at $n=8,6$, and 3 for $\sigma_{e}^{2}=9,4$, and 1 , respectively $\left(\sigma_{e}^{2}\right.$ given in units of $\sigma_{m}^{2}$ ). Thus weaker selection produced greater genetic gains. Similar results were obtained by Davies (1964) in a simulation study, and have been reported in practice by Calam (1964). This is due to the increase in the genetic standard deviation, $\sigma_{g(t)}$ with increased $n$.

The main lesson of table 2 is that at optimum $n$, measurement error has little effect on expected genetic gains, while increased $\mathcal{N}$ will increase gains up to two-fold. Thus the results in table 2 emphasise that in selection programmes of this sort the prime determinant of genetic gains will be the absolute value of the induced genetic variance, $\sigma_{m}^{2}$. Error variance, $\sigma_{e}^{2}$, as it affects the correlation between genotype and phenotype, and testing facilities, $\mathcal{N}$, as they affect the selection differential, $i$, will be of secondary importance, but are by no means negligible. 
The values of table 2 give net genetic gains per cycle in the absence of any deleterious effect of mutagenesis on the population mean $(d=0)$. If a deleterious effect is present, net gains will be decreased more or less greatly in proportion to $d$, and can even be negative. This was apparently the situation for the distribution studied in Davies' (1964) simulation study. As his results clearly show, in such circumstances optimum design can make all the difference between success and failure of the selection programme. More than this, if the effect of mutagenesis on mean population value is strong enough $d$ will override $\Delta G_{m}$, and for given $\mathcal{N}$ and $\sigma_{e}^{2}$, even optimum programmes may result in decreased yields. For example, if $d=-1.5 \sigma_{m}$, table 2 shows that for $\mathcal{N}=50$, progress would be negative, for $\mathcal{N}=200$ progress would be negligible, while for $\mathcal{N}=1000$ significant progress might be obtained. Thus, for such cases it will be necessary to devote additional effort to the selection programme by increasing $\mathcal{N}$ and decreasing $\sigma_{e}^{2}$, or devise suitable selective screening procedures to increase $\sigma_{m}^{2}$ and reduce $d$. (Dulaney and Dulaney, 1967; Demain, 1972; Elander, 1976.)

\section{(ii) Response to selection under recurrent cycles of mutagenesis and selection} followed by recurrent cycles of hybridisation and selection

Table 3 shows the relative progress expected by a combined programme of mutational selection cycles followed by recombinational selection cycles. Progress during the recombinational cycles relative to the mutational cycles within each line, and overall progress in the combined programme relative to that given by mutagenesis alone, increased with decreasing error variance

\section{TABLE 3}

Mean expected gains per cycle of hybridisation and selection for a quantitative trait in a selection programme based on $t_{m}$ cycles of mutagenesis and selection followed by $t_{r}$ cycles of hybridisation and selection, according to error variance.* Gains in the hybridisation cycles $\left(\Delta G_{r}\right)$ shown relative to gains in the preceding mutagenesis cycles $\left(\Delta G_{m}\right)$, and gains in the combined mutagenesis-hybridisation programme $\left(\Delta G_{c}\right)$ shown relative to gains in a programme based on mutagenesis alone $\left(\Delta G_{m}^{\prime}\right) \dagger$

\begin{tabular}{|c|c|c|c|c|c|c|c|c|c|}
\hline \multirow[b]{2}{*}{$\begin{array}{l}\text { Number of } \\
\text { mutational } \\
\text { cycles }\left(t_{m}\right)\end{array}$} & \multicolumn{3}{|c|}{$\sigma_{e}^{2}=9 \cdot 0$} & \multicolumn{3}{|c|}{$\sigma_{e}^{2}=4 \cdot 0$} & \multicolumn{3}{|c|}{$\sigma_{e}^{2}=1 \cdot 0$} \\
\hline & $t_{r}$ & $\frac{\Delta G_{r}}{\Delta G_{m}}$ & $\frac{\Delta G_{c}}{\Delta G_{m}^{\prime}}$ & $t_{r}$ & $\frac{\Delta G_{r}}{\Delta G_{m}}$ & $\frac{\Delta G_{c}}{\Delta G_{m}^{\prime}}$ & $t_{r}$ & $\frac{\Delta G_{r}}{\Delta G_{m}}$ & $\frac{\Delta G_{c}}{\Delta G_{m}^{\prime}}$ \\
\hline 1 & $0_{+}^{+}$ & $1 \cdot 00_{+}^{+}$ & $0.88_{+}^{+}$ & $0 \ddagger$ & $1 \cdot 00_{+}^{+}$ & $0.88_{+}^{+}$ & $0_{+}^{+}$ & $1 \cdot 00_{+}^{+}$ & $0.88_{+}^{+}$ \\
\hline 2 & $0 \ddagger$ & $1.00 \ddagger$ & $0.88 \mp$ & 1 & 1.27 & 0.96 & 1 & 1.95 & $1 \cdot 18$ \\
\hline 3 & 2 & $1 \cdot 19$ & 0.93 & 2 & $1 \cdot 50$ & $1 \cdot 06$ & 1 & $2 \cdot 48$ & $1 \cdot 22$ \\
\hline 4 & 2 & $1 \cdot 54$ & $1 \cdot 01$ & 2 & 1.91 & $1 \cdot 15$ & 1 & $2 \cdot 92$ & $1 \cdot 24$ \\
\hline 5 & 2 & $1 \cdot 86$ & 1.07 & 2 & $2 \cdot 28$ & $1 \cdot 20$ & 2 & $2 \cdot 50$ & $1 \cdot 28$ \\
\hline 6 & 2 & $2 \cdot 16$ & $1 \cdot 11$ & 2 & $2 \cdot 62$ & $1 \cdot 24$ & 2 & $3 \cdot 00$ & $1 \cdot 34$ \\
\hline 7 & 3 & $2 \cdot 14$ & $1 \cdot 15$ & 2 & 2.93 & $1 \cdot 26$ & 2 & $3 \cdot 65$ & $1 \cdot 42$ \\
\hline 8 & 3 & $2 \cdot 40$ & $1 \cdot 19$ & 2 & $3 \cdot 22$ & 1.27 & 2 & 3.97 & $1 \cdot 42$ \\
\hline 9 & 3 & $3 \cdot 46$ & $1 \cdot 22$ & 3 & $2 \cdot 99$ & $1 \cdot 32$ & 2 & $4 \cdot 26$ & $1 \cdot 42$ \\
\hline 10 & 3 & $4 \cdot 05$ & $1 \cdot 24$ & 3 & $3 \cdot 27$ & $1 \cdot 34$ & 2 & $4 \cdot 53$ & $1 \cdot 42$ \\
\hline
\end{tabular}

* Error variance in units of the variance induced by mutagenesis.

† Combined programme involves two selection lines each based on the best 5 colonies out of 100 tested. Mutagenesis only, involves one selection line based on the best 5 out of 200 colonies tested. See text for additional details.

+ For this $t_{m}$ hybridisation cycles were omitted from the combined programme since gains in the hybridisation cycles of the combined programme were expected to be less than those in the preceding mutagenesis cycles. See text for additional details. 
and with increased number of mutational cycles preceding the recombinational cycles. Small deviations from these trends (e.g., in the case of $t_{m}=9$, $\sigma_{e}^{2}=4.0$ ) occasionally occur as a result of the stepwise increase in $t_{r}$ at these points. At the selection intensities postulated $(i=2 \cdot 0)$, two or three recombinational cycles suffice to exhaust recombinational variability almost completely and to signal the initiation of a new series of mutational cycles. Trial calculations based on expression (4) and using the results of table 3 to estimate $t_{r}$, show that dividing available resources among more than two lines does not yield any appreciable increase in the rate of progress. The reason for this lies in the strong reduction in $a_{r}$ compared to $a_{m}$ in this case, and in the increase in $t_{r}$ due to the additional generations of hybridisation and selection required in order to collect favourable alleles from all lines together in a single line.

\section{Discussion}

(i) Effect of deviations from the assumptions

(a) Non-normality of the genetic variation induced by mutagenesis

The calculations of table 1 are based on the assumption that the genetic variation induced by mutagenesis is normally distributed. This appears to be the situation, at least roughly, in some cases (Alikhanian, 1970; Dulaney and Dulaney, 1967). If the distribution of genetic variation is not normal, and particularly if it is skewed in the direction of lower values (as was the distribution studied by Davies, 1964), heritabilities and genetic variance at the positive and negative tails of the post-mutagenic phenotypic distribution will differ. In this case it is really the genetic variance at the positive tail of the post-mutagenic distribution that determines genetic progress. In the extreme case, where all mutations have a negative effect, heritabilities on the positive tail of the post-mutagenic distribution will equal zero and progress will be nil. In less extreme cases, when some mutations do have positive effects, the effects of non-normality on heritabilities on the positive side of the post-mutagenic distribution are not drastic (Nishida and Abe, 1974). Furthermore, the effects will tend to decrease with increased cycles of mutagenesis and selection, since that portion of the genetic variation that derives from genetic differences between the colonies selected in the previous generation (i.e., $\sigma_{w}^{2}$ ) is normally distributed and will generally be large relative to $\sigma_{m}^{2}$ unless $\sigma_{e}^{2}$ and $n$ are small (table 1). Thus the major effect of moderate non-normality is to make heritabilities on the high side of the phenotypic distribution less than they would be, considering the entire range of induced genetic variation.

\section{(b) Genotype $\times$ environment interactions}

In the calculations of table 2, genetic gains were accumulated from generation to generation on the assumption that genotype $\times$ test-environment interactions were absent. This implies that a given mutation will have more or less the same effect over the usual run of random environmental fluctuations differentiating successive series of tests. This seems a fairly reasonable assumption, but it does not always hold, particularly in scaling up operations (Burnett, 1975, pp. 140, 243). When it does not, that part of $\sigma_{m}^{2}$ attributable to genotype $\times$ test-environment interaction moves 
from the $\sigma_{m}^{2}$ term to $\sigma_{e}^{2}$, simultaneously reducing $\sigma_{m}^{2}$ and $h_{t}^{2}$. As a consequence cumulated genetic gains over a number of cycles can be much less than expected on the basis of within cycle calculations (Dickerson, 1955). One possible solution is to replicate tests over time, but this can seriously reduce the rate of progress.

\section{(c) Genetic drift, overlapping mutations and epistasis}

The results of table 3 are based on the assumptions of non-overlapping mutations and no loss of favourable alleles by genetic drift during the recombinational cycles. Considering the small number of parental colonies chosen during the recombinational cycles, however, one would expect a small but not insignificant number of favourable alleles to be lost by drift, with a corresponding reduction in genetic gains. A more severe restriction on the gain expected from the recombinational cycles is the assumption that the mutations in the two mutational lines are non-overlapping. For moderate degrees of overlapping the effect on relative progress would not be too great, e.g., using expression (4) we find that for $v=0 \cdot 20$, the additional gains given by the combined programme compared to mutagenesis alone would be decreased by 30 per cent. For higher degrees of overlap, gains would become negligible or even negative. After each round of mutational and recombinational cycles, the number of loci remaining that can undergo positive mutation is expected to decrease by $t_{m}(2-v)$. This may be an overestimate, however. Some loci may be able to undergo repeated positive mutations, reaching a higher quantitative value each time. Also, as the genome is remoulded by the accumulation of plus alleles at many loci, loci that previously had little effect on the trait of interest may take on increased importance (Hostalek and Vanek, 1973; Calam, Daglish and McCann, 1976). As a result the decrease in potentially mutable loci with repeated rounds of mutation and recombination may be slower than expected.

The results of table 3 are also based on the assumption that epistasis is absent. If successive mutations affecting a quantitative trait build upon epistatic interactions with the mutations that have come before, the first recombinational cycle would exhibit a decrease in mean value due to the breakup of favourable epistatic combinations. In this case, loss of alleles by genetic drift during the recombinational cycles and overlapping mutations could be of greater consequence. Loss of critical alleles by drift could prevent the reconstitution of favourable epistatic combinations. The same would be true if epistatic combinations in the two lines involved alternative alleles at one or more loci. Both effects might considerably reduce the final gains realised through hybridisation.

\section{(ii) Hybridisation compared to mutagenesis alone}

The results of this study show that the anticipated return of a decision to divide test facilities among two mutagenic selection lines, with the intention of crossing them at a future date, increases with the number of loci potentially capable of mutating to plus alleles, and with the relative magnitude of $\sigma_{m}^{2}$ as compared to $\sigma_{e}^{2}$. These requirements seem contradictory in practice. When the variance induced by mutagenesis is large (as in selecting for yield of primary metabolites using a specific selective screen), 
the number of loci potentially affected is likely to be small; and when the number of potential loci is large (as in mass screening for primary or secondary metabolites), the variance induced by mutation is likely to be small relative to error variance. Thus, the analysis suggests that within a single testing programme the utility of dividing available resources among two selection lines is likely to be marginal, and perhaps negative unless, and it is an important unless, mutagenesis has a deleterious effect on mean population value. In this case as pointed out in section 2.2 .1 hybridisation can make a major contribution by allowing additional plus alleles to be incorporated into the line without the necessity for additional mutagenesis. In addition it may be possible, during the recombinational cycles, to eliminate many of the deleterious alleles contributing to negative $d$ values. This would be an example of the usefulness of recombination in avoiding Muller's " ratchet effect" as discussed by Felsenstein (1974). It should also be emphasised that under most circumstances, it would be potentially useful to cross selection lines obtained in independent testing programmes (Calam, Daglish and McCann, 1976).

\section{(iii) Applied aspects}

\section{(a) Parameter estimation}

Estimates of the effect of mutagenesis on the mean and standard deviation of a mutagenised population can be obtained by comparing the postmutagenic distribution of phenotypic value in a population of mutagenised colonies with that of a population of non-mutagenised colonies, both from the same source. Genotype $\times$ test-environment interactions could be estimated in the same experiment by reculturing and retesting the colonies and comparing results in the two series of tests. Thus, on the basis of relatively small-scale preliminary observations, the model presented here could allow alternative breeding programmes to be compared with respect to expected genetic progress. This could be particularly important in deciding on the facilities to allot to any particular programme since the marginal contribution of additional testing facilities to genetic progress depends critically on the effect of mutagenesis on the mean value of the post-mutagenic population, as discussed in the results section.

It is of interest to consider the extent to which experimental or applied situations can be found in which the model presented here could make some contribution to the design of selection programmes. Caten and Jinks (1976) and Burnett (1975) have summarised experimental work on quantitative traits in fungi, showing that quantitative traits in these organisms do act in accordance with biometrical expectations so far as naturally occurring quantitative variation is concerned. On this basis, it seems reasonable to expect biometric models to provide useful guides for the design of experimental selection under mutagenesis in such organisms, as proposed by Merrick (1976) and found to be the case by Simpson and Caten (unpublished data, cited in Hopwood and Merrick, 1977). In industrial selection programmes, those involving primary metabolites are generally based on highly selective screening techniques that provide post-mutagenic populations heavily enriched in high-yielding mutants (von Borstel and Mehta, 1976). For such situations, $d$ will be large and positive and the model developed here would not be useful. One suspects, however, that continued improve- 
ment in such situations would eventually exhaust the possibilities inherent in loci amenable to selective screening techniques. At this point, a less specific, mass screening approach, drawing on a broader group of loci might provide additional gains. For such programmes, $\sigma_{m}^{2}$ and $\sigma_{e}^{2}$ might be of comparable magnitudes, and questions of optimal programme design would increase in importance. Similarly, highly specific screening techniques for secondary metabolites (e.g., antibiotics) are generally not as yet available (Alikhanian, 1970; Elander, 1976) although methods for partial enrichment have been described (Dulaney and Dulaney, 1967; Demain, 1972; Woodruff, 1973). In such situations, particularly in the initial stages of a screening programme, application of the model presented here might be of some assistance in optimising the programme with respect to available facilities, and in deciding on the overall scale of effort to invest in the improvement programme.

Acknowledgments.- The author acknowledges with thanks the contributions of B. Dorfman and A. Genizi to the formulation and clarification of this model. This research was supported by Grant No. 226 of the U.S.-Israel Binational Research Foundation.

\section{REFERENCES}

alikhanian, s. 1970. Applied aspects of microbial genetics. Current Topics in Microbiol. and Immunol., 53, 91-148.

BeCKer, w. 1967. Manual of Procedures in Quantitative Genetics. Washington State University Press, Pullman, Washington.

BURNETT, J. H. 1975. Mycogenetics. John Wiley and Sons, London.

CALAM, C. T. 1964. The selection, improvement and preservation of microorganisms. Prog. Indust. Microbiol., 5, 1-53.

CAlam, C. T., DAGLiSh, L. B., AND MCCANN, E. P. 1976. Penicillin: tactics in strain improvement. In: Genetics of Industrial Microorganisms, ed. K. D. Macdonald. Academic Press, New York, pp. 273-287.

CATEN, C. E., AND JINKs, J. L. 1976. Quantitative genetics. In: Genetics of Industrial Microorganisms, ed. K. D. MacDonald. Academic Press, New York, pp. 93-111.

DAvies, o. L. 1964. Screening for improved mutants in antibiotic research. Biometrics, 20, 576-591.

DEMAIN, A. L. 1972. Genetics and process improvement in Streptomyces. Fermentation Technology Today. Society of Fermentation Technology, Japan, pp. 239-246.

Dickerson, G. E. 1955. Genetic slippage in response to selection for multiple objectives. Cold Spr. Harb. Symp. Quant. Biol., 20, 213-224.

DUlaney, E. L., AND DULANEY, D. D. 1967. Mutant populations of Streptomyces viridifaciens. Trans. N.Y. Acad. Sci., 29, 782-799.

ELANDER, R. P. 1976. Mutation to increased product formation in antibiotic-producing microorganisms. In: Microbiology, 1976, ed. D. Schlessinger. Amer. Soc. Microbiol., Wash. D.C., pp. 507-509.

FALCONER, D. s. 1960. Introduction to Quantitative Genetics. The Ronald Press Co., New York.

Felsenstein, J. 1974. The evolutionary advantage of recombination. Genetics, 78, 737-756. HOPWOOD, D. A., AND MERRICK, M. J. 1977. Genetics of antibiotic production. Bacteriological Reviews, 41, 593-635.

hostaleK, z., AND VANEK, z. 1973. Molecular basis of polygenic inheritance in the biosynthesis of chlortetracycline. In: Genetics of Industrial Microorganisms, Vol. 2, eds. Z. Vanek, Z. Hostalek and J. Cudlin. Elsevier Publ. Co., Amsterdam, pp. 265-282.

MERRICK, M. J. 1976. Hybridisation and selection for penicillin production in Aspergilus nidulans-a biometrical approach to strain improvement. In: Genetics of Industrial Microorganisms, ed. K. D. MacDonald. Academic Press, New York, pp. 229-242.

NISHIDA, A., AND ABE, T. 1974. The distribution of genetic and environmental effects and the linearity of heritability. Canad. F. of Genetics and Cytology, 16, 3-10. 
SERMONTI, G. 1969. The genetics of antibiotic producing microorganisms. John Wiley and Sons, Inc., New York.

SOLLER, M., AND GENIZi, A. 1975. A note on assortative mating and artificial selection. Heredity, 34, 435-437.

von BORSTEL, R. C., AND MEHTA, R. D. 1976. Mutation and selection systems for yeasts. In: Microbiology, 1976, ed. D. Schlessinger. Amer. Soc. Microbiol., Wash., D.C., pp. 507-509.

WOODRUFF, H. B. 1973. Antibiotic production as an aspect of secondary metabolism. In: Genetics of Industrial Microorganisms, Vol. 2, ed. Z. Vanek, Z. Hostaliek and J. Cudlin. Elsevier Publ. Co., Amsterdam, pp. 265-282. 